\title{
BMJ Open Cost-effectiveness of enhanced recovery in hip and knee replacement: a systematic review protocol
}

\author{
Jacqueline Murphy, ${ }^{1}$ Mark G Pritchard, ${ }^{1,2}$ Lok Yin Cheng, ${ }^{1}$ Roshni Janarthanan, ${ }^{1}$ \\ José Leal ${ }^{1}$
}

To cite: Murphy J,

Pritchard MG, Cheng LY, et al. Cost-effectiveness of enhanced recovery in hip and knee replacement: a systematic review protocol. BMJ Open 2018;8:e019740. doi:10.1136/ bmjopen-2017-019740

- Prepublication history and additional material for this paper are available online. To view these files, please visit the journal online (http://dx.doi. org/10.1136/bmjopen-2017019740).

Received 22 September 2017 Revised 10 November 2017 Accepted 15 December 2017

Check for updates

${ }^{1}$ Health Economics Research Centre, Nuffield Department of Population Health, Medical Sciences Division, University of Oxford, Oxford, UK

${ }^{2}$ Oxford University Hospitals NHS Foundation Trust, John Radcliffe Hospital, Oxford, UK

Correspondence to

Dr José Leal;

jose.leal@ndph.ox.ac.uk

\section{ABSTRACT}

Introduction Hip and knee replacement represents a significant burden to the UK healthcare system. 'Enhanced recovery' pathways have been introduced in the National Health Service (NHS) for patients undergoing hip and knee replacement, with the aim of improving outcomes and timely recovery after surgery. To support policymaking, there is a need to evaluate the cost-effectiveness of enhanced recovery pathways across jurisdictions. Our aim is to systematically summarise the published costeffectiveness evidence on enhanced recovery in hip and knee replacement, both as a whole and for each of the various components of enhanced recovery pathways. Methods and analysis A systematic review will be conducted using MEDLINE, EMBASE, Econlit and the National Health Service Economic Evaluations Database. Separate search strategies were developed for each database including terms relating to hip and knee replacement/arthroplasty, economic evaluations, decision modelling and quality of life measures. We will extract peer-reviewed studies published between 2000 and 2017 reporting economic evaluations of preoperative, perioperative or postoperative enhanced recovery interventions within hip or knee replacement. Economic evaluations alongside cohort studies or based on decision models will be included. Only studies with patients undergoing elective replacement surgery of the hip or knee will be included. Data will be extracted using a predefined pro forma following best practice guidelines for economic evaluation, decision modelling and model validation. Our primary outcome will be the cost-effectiveness of enhanced recovery (entire pathway and individual components) in terms of incremental cost per quality-adjusted life year. A narrative synthesis of all studies will be presented, focussing on cost-effectiveness results, study design, quality and validation status.

Ethics and dissemination This systematic review is exempted from ethics approval because the work is carried out on published documents. The results of the review will be disseminated in a peer-reviewed academic journal and at conferences.

PROSPERO registration number CRD42017059473.

\section{INTRODUCTION}

Hip and knee replacement represents a significant burden to the UK healthcare system. In 2015, over 88000 primary total

\section{Strengths and limitations of this study}

- This systematic review of enhanced recovery pathway for hip/knee replacement will be based on a detailed search strategy that will be complemented with a comprehensive data extraction and analysis of the studies.

- The review will follow the latest guidelines and the quality and validity of the cost-effectiveness evidence will be assessed using published modelling checklists (modelling specific or general economic evaluation checklists as appropriate).

- The quality and validity of the evidence may depend on the reporting quality and transparency of the identified studies.

hip replacements (THRs) and primary total knee replacements (TKRs) were registered in the National Joint Registry, covering procedures performed in National Health Service (NHS) and independent hospitals in England, Wales, Northern Ireland and the Isle of Man. ${ }^{12}$

Following the establishment of the Department of Health Enhanced Recovery Partnership Programme in April 2009 ${ }^{3}$ a new 'enhanced recovery' pathway has been introduced in many NHS hospitals for patients undergoing hip and knee replacement. ${ }^{4}$ According to the Department of Health report, ${ }^{3}$ the principles of enhanced recovery are to ensure "the patient is in the best possible condition for surgery; the patient has the best possible management during and after his/ her operation; the patient experiences the best postoperative rehabilitation'. Therefore, enhanced recovery considers the preoperative, perioperative and postoperative management of patient care to enable improved and faster recovery and discharge from hospital. Enhanced recovery programmes vary between hospitals, but generally include a combination of best practice initiatives and medical interventions. Examples of such 
interventions include (preoperative) patient education and setting of expectations around surgery and rehabilitation, nutrition, physiotherapy; (perioperative) optimised anaesthesia, shortened surgical times, minimal use of drains and tubes; (postoperative) same day mobilisation and discharge, engagement of multidisciplinary teams in provision of physiotherapy and occupational therapy, clear rehabilitation instructions and/or other interventions as agreed in each hospital.

To inform national policy and local decisions across many jurisdictions, evidence on both the effectiveness and cost-effectiveness of interventions is needed. Economic evaluations of enhanced recovery interventions in hip and knee replacement patients provide such evidence. Estimates of the impact of the interventions in terms of quality of life and costs relative to current practice enable providers to base decisions not only on clinical effectiveness but also on their value for money.

Previous systematic reviews of economic evaluations in patients having hip or knee replacement have not looked at enhanced recovery or its components but rather focused on the surgical procedure and its cost-effectiveness. $^{5-9}$ This recent evidence suggests that total joint replacement is cost-effective compared with conservative management, ${ }^{57}$ and unicompartmental knee replacement is less costly than TKR and for some age groups is more effective. ${ }^{6}$ We therefore identified a need for a more comprehensive summary of the published economic evidence on enhanced recovery in hip and knee replacement, including each component of the pathway from preoperative to postdischarge.

Our aim is to systematically summarise and assess the quality of cost-effectiveness evidence of enhanced recovery in hip and knee replacement, for patients of any age with common indications for surgery. Our objectives are to:

- Summarise peer-reviewed published economic evaluations of enhanced recovery interventions in populations of individuals undergoing elective hip or knee replacement.

- Report the cost-effectiveness findings in terms of cost per quality-adjusted life year (QALY) gained for the overall pathway and individual components of enhanced recovery (preoperative, perioperative and postoperative interventions).

- Assess study quality and risk of bias.

- Identify and discuss research gaps for future economic evaluations.

\section{METHODS AND ANALYSIS}

\section{Review registration and timelines}

This systematic review protocol has been developed with reference to the Preferred Reporting Items for Systematic Review and Meta-Analysis Protocols 2015 (PRISMA-P) guidelines ${ }^{10}$ and published recommendations for performing systematic reviews of economic evaluations. ${ }^{11-13}$ The systematic review is registered in the International Prospective Register of Systematic Reviews
(PROSPERO), registration number CRD42017059473. ${ }^{14}$ Important amendments to this protocol will be reported and published with the results of the review.

\section{Search strategy}

We have defined the search strategies and database selection with assistance from an information specialist and by comparing our search terms with those from previous reviews and review protocols of economic evaluations in hip/knee replacement. ${ }^{5-9}$

The following electronic databases will be searched up to 1 March 2017 (with no start date specified): Ovid MEDLINE, Embase, the NHS Economic Evaluations Database (NHS EED) (via the Cochrane Library) and EconLit (via ProQuest). NHS EED contains records of economic evaluations published up until the end of December 2014, with bibliographic records being added to the database up to March 2015. ${ }^{15}$ We anticipate that economic studies published after December 2014 will be identified using the other databases in the review.

Articles will be restricted to English-language literature but no geographical restrictions will be applied to the search. Abstracts or conference presentations will not be included as results are not presented in sufficient detail to allow critical appraisal of the economic evaluations. Date restrictions limiting the review to studies published after the year 2000 will be applied during the study selection process.

The search strategy and inclusion/exclusion criteria were piloted by two reviewers. For the latter, the search was run and inclusion/exclusion criteria were applied to $10 \%$ of the search results to check consistency between reviewers. The search strategies include terms relating to hip and knee replacement, economic evaluations, decision modelling and quality of life measures. The full search strategies are provided in the online supplementary file 1. Additional articles will be identified by searching the reference list of the studies included in this review as well as those of previous literature reviews on economic evaluations of hip or knee replacement populations.

\section{Study selection}

ENDNOTE X7, Thomson Reuters, will be used to manage the references. Duplicates will be removed after the initial searches and two reviewers will independently assess all abstracts to determine whether a full text review is needed. Discrepancies will be resolved between reviewers or referred to a third study team member. Following PRISMA guidelines, we will present a flow diagram reporting the selection process.

\section{Study eligibility criteria}

\section{Population}

We will include studies with participants undergoing THR and TKR surgery for common indications. In the UK, osteoarthritis was the surgical indication in $90 \%$ of primary hip replacement procedures ${ }^{16}$ and $96.1 \%$ of primary knee replacement procedures in $2015 .{ }^{17}$ We will 
therefore include studies with osteoarthritis as an indication for surgery, though we do not intend to prespecify a minimum required proportion of patients with this indication. Studies exclusively concerning populations with other indications such as avascular necrosis, inflammatory arthropathy, previous/failed surgery, cancer, congenital conditions or infection will be excluded, as will studies looking at emergency procedures (eg, due to trauma).

\section{Intervention}

Economic evaluations of any preoperative, perioperative or postoperative intervention within the hip/knee replacement enhanced recovery pathway will be included, in addition to studies considering enhanced recovery pathways as a whole. Interventions must be those that form part of the usual pathway of care (with or without enhanced recovery) for hip/knee replacement.

\section{Comparators}

The comparator in each study must be an intervention within the clinical pathway of hip or knee replacement, respectively, or no intervention/placebo. Studies with comparators consisting only of interventions not within the hip or knee replacement pathway (eg, comparing to non-surgical interventions) will be excluded.

\section{Types of studies}

Both model-based evaluation and randomised controlled trials/cohort-based economic evaluation will be included. We will restrict the analysis to cost-utility analyses (ie, reporting costs per QALY gained) but will report number of cost-effectiveness studies giving incremental costs per other units of health gain (eg, life years). As cost-utility analysis is the preferred approach to inform decisions on healthcare resource allocation, ${ }^{18}{ }^{19}$ we will also exclude cost-benefit analysis and cost-minimisation analysis, as well as cost-consequence analysis if incremental costs per QALYs gained cannot be estimated from the results.

\section{Outcomes}

In order to inform policy and extract comparable data between studies, the primary outcome of interest is cost-effectiveness findings in terms of the incremental cost per QALY gained. In addition, we will report the absolute costs and QALYs per intervention being evaluated as well as the respective incremental values relative to current care.

The secondary outcomes of interest are the probability of being the most cost-effective intervention (to reflect uncertainty), value of information (VoI) if reported, study design and quality, model type, structure and validation status (for model-based studies) and the source and quality of the data used for the analysis.

\section{Data extraction}

Data extraction will be divided between two reviewers using a standardised form and referred to a third reviewer where necessary to resolve discrepancies. Data extraction items are based on published checklists ${ }^{20-24}$ and will include study question and comparators, patient population, study type (model or trial-based economic evaluation), model type and design (where applicable), data sources and hierarchy of evidence (quality assessment), currency and cost year, cost-effectiveness results (point estimate and probability of being cost-effective), VoI results (if reported), study conclusions and a risk of bias assessment. The pro forma for data extraction is given in the online supplementary file 2. Extracted data will be entered into a spreadsheet in Microsoft Excel and the completed data extraction form for each study will be retained. The data extraction forms have been piloted by two reviewers using selected examples of included studies.

\section{Risk of bias}

In line with published recommendations, ${ }^{13}$ the quality of reporting and risk of bias of the economic evaluations will be assessed using published checklists from the Consensus on Health Economic Criteria project ${ }^{25}$ for economic evaluations and the International Society for Pharmacoeconomics and Outcomes Research taskforce for decision models $^{26}$ (see online supplementary file 3 ). Items in the checklists will be marked as Yes, No, Unknown or Not Applicable for each study, and a final assessment of the risk of bias will be made by the reviewer.

\section{Data synthesis}

Data for synthesis will be managed using Microsoft Excel. A narrative synthesis will be presented outlining the overall cost-effectiveness findings from the included studies. Hip and knee replacement findings will be reported separately. We will also discuss the quality and risk of bias of the individual studies and the generalisability of the findings to settings other than those reported, in order to assess the overall strength of the body of economic evidence. Using the results of sensitivity analyses and VoI methods (if available), we will report recommendations for further research to reduce decision uncertainty. Finally, we will identify any gaps in the evidence that need to be addressed in future evaluations of recovery pathway interventions in populations of hip or knee replacement patients.

\section{DISCUSSION}

Cost-utility data are relevant to understand the value of healthcare interventions and to support decisions concerning which interventions to implement in jurisdictions where healthcare resources are limited. Given the high volume of hip and knee replacement and the associated costs, there is significant interest in identifying cost-effective strategies to improve the recovery time of these patients. We anticipate that the review will influence practice by providing a comprehensive summary of the cost-effectiveness of enhanced recovery components according to measures that are comparable between interventions. This will enable healthcare providers to tailor their approach according to the most cost-effective 
interventions. Our findings will inform the challenges and research gaps concerning future economic evaluations of enhanced recovery interventions. We anticipate that this review may also inform future guidelines around enhanced recovery by providing robust cost-effectiveness evidence from international studies.

\section{Ethics and dissemination}

This systematic review is exempt from ethics approval and consent to participate because the work is carried out on published documents. The results of the review will be disseminated in a peer-reviewed academic journal and at conferences.

Acknowledgements The authors would like to thank Nia Roberts, information specialist at the University of Oxford, for assistance with the search strategy and database selection and Rafael Pinedo-Villanueva, Health Economist at the Nuffield Department of Orthopaedics, Rheumatology and Musculoskeletal Sciences, University of $0 x$ ford, for assistance with the search strategy.

Contributors JM, LYC and JL developed the search strategies. JM and LYC piloted the search strategy and inclusion criteria. JM and JL developed the data extraction pro forma. JM, MGP, LYC and RJ piloted the data extraction pro-forma. JL is the guarantor of the review. All authors defined the inclusion criteria and approved the final manuscript.

Funding This project was funded by the National Institute for Health Research Health Services and Delivery Research Programme (project number 14/46/02). Support was received from the Oxford NIHR Biomedical Research Centre, Nuffield Orthopaedic Centre, University of Oxford. The views and opinions expressed therein are those of the authors and do not necessarily reflect those of the HS\&DR Programme, NIHR, NHS or the Department of Health.

Competing interests None declared.

Patient consent Not required.

Provenance and peer review Not commissioned; externally peer reviewed.

Open Access This is an Open Access article distributed in accordance with the Creative Commons Attribution Non Commercial (CC BY-NC 4.0) license, which permits others to distribute, remix, adapt, build upon this work non-commercially, and license their derivative works on different terms, provided the original work is properly cited and the use is non-commercial. See: http://creativecommons.org/ licenses/by-nc/4.0/

(c) Article author(s) (or their employer(s) unless otherwise stated in the text of the article) 2018. All rights reserved. No commercial use is permitted unless otherwise expressly granted.

\section{REFERENCES}

1. National Joint Registry. Secondary 13th annual report (online statistics): procedure details by type of provider (hip). $2016 \mathrm{http}: / /$ www.njrreports.org.uk/hips-all-procedures-activity (accessed 12 Dec 2016).

2. National Joint Registry. Secondary 13th annual report (online statistics): procedure details by type of provider (knee). $2016 \mathrm{http}: / /$ www.njrreports.org.uk/knees-all-procedures-activity (accessed 12 Dec 2016).

3. Department of Health. Enhanced Recovery Partnership Project Report - March 2011: Department of Health, NHS Improvement NCAT, NHS Institute, 2011.

4. Enhanced Recovery Partnership Programme. Delivering enhanced recovery - helping patients to get better sooner after surgery Department of Health, 2010.

5. Nwachukwu BU, Bozic KJ, Schairer WW, et al. Current status of cost utility analyses in total joint arthroplasty: a systematic review. Clin Orthop Relat Res 2015;473:1815-27.

6. Burn E, Liddle AD, Hamilton TW, et al. Choosing between unicompartmental and total knee replacement: what can economic evaluations tell us? A systematic review. Pharmacoecon Open 2017;1:241-53.
7. Daigle ME, Weinstein AM, Katz JN, et al. The cost-effectiveness of total joint arthroplasty: a systematic review of published literature. Best Pract Res Clin Rheumatol 2012;26:649-58.

8. Bozic KJ, Saleh KJ, Rosenberg AG, et al. Economic evaluation in total hip arthroplasty: analysis and review of the literature. $J$ Arthroplasty 2004;19:180-9.

9. Saleh KJ, Gafni A, Saleh L, et al. Economic evaluations in the hip arthroplasty literature: lessons to be learned. J Arthroplasty 1999:14:527-32.

10. Moher D, Shamseer L, Clarke M, et al. Preferred reporting items for systematic review and meta-analysis protocols (PRISMA-P) 2015 statement. Syst Rev 2015;4:1.

11. Thielen FW, Van Mastrigt G, Burgers LT, et al. How to prepare a systematic review of economic evaluations for clinical practice guidelines: database selection and search strategy development (part 2/3). Expert Rev Pharmacoecon Outcomes Res 2016:16:705-21.

12. van Mastrigt GA, Hiligsmann M, Arts $\mathrm{JJ}$, et al. How to prepare a systematic review of economic evaluations for informing evidencebased healthcare decisions: a five-step approach (part 1/3). Expert Rev Pharmacoecon Outcomes Res 2016;16:689-704.

13. Wijnen B, Van Mastrigt G, Redekop WK, et al. How to prepare a systematic review of economic evaluations for informing evidencebased healthcare decisions: data extraction, risk of bias, and transferability (part 3/3). Expert Rev Pharmacoecon Outcomes Res 2016;16:723-32.

14. Murphy J, Cheng LY, Leal J. Cost-effectiveness and quality of life of enhanced recovery in hip and knee replacement: a systematic review protocol. http://www.crd.york.ac.uk/PROSPERO/display_record.asp? ID=CRD42017059473

15. Centre for Reviews and Dissemination. News archive: changes to DARE and NHS EED (news item 14 January 2015). Secondary news archive: changes to DARE and NHS EED (news item 14 January 2015). www.crd.york.ac.uk/crdweb/newspage.asp\#htadatabase (accessed 30 Mar 2017).

16. National Joint Registry. 13th annual report (online statistics): patient characteristics for primary hip replacement procedures in 2015, according to procedure type. Secondary 13 th annual report (online statistics): patient characteristics for primary hip replacement procedures in 2015 , according to procedure type. http://www. njrreports.org.uk/hips-primary-procedures-patient-characteristics (accessed 18 Jan 2017).

17. National Joint Registry. Secondary 13th annual report (online statistics): Patient characteristics for primary knee replacement procedures in 2015, according to procedure type. http://www. njrreports.org.uk/knees-primary-procedures-patient-characteristics (accessed 18 Jan 2017).

18. National Institute for Health and Care Excellence. Guide to the methods of technology appraisal: Chapter 5 The reference case [PMG9, 2013.

19. Gold MR, Siegel JE, Russell LB, et al. Cost-effectiveness in health and medicine: Oxford University Press, 1996.

20. Drummond MF, Jefferson TO. Guidelines for authors and peer reviewers of economic submissions to the BMJ. The BMJ Economic Evaluation Working Party. BMJ 1996;313:275-83.

21. Husereau D, Drummond M, Petrou S, et al. Consolidated Health Economic Evaluation Reporting Standards (CHEERS) statement. BMJ 2013;346:f1049.

22. Coyle DL KM. Chapter 5: Evidence-based economic evaluation: how the use of different data sources can impact results. In: Donaldson CMM, Vale L, eds. Evidence-based health economics: from effectiveness to efficiency in systematic review: BMJ Publishing Group, 2002.

23. Vemer P, Corro Ramos I, van Voorn GA, et al. AdViSHE: A validationassessment tool of health-economic models for decision makers and model users. Pharmacoeconomics 2016;34:349-61.

24. Philips Z, Bojke L, Sculpher M, et al. Good practice guidelines for decision-analytic modelling in health technology assessment: a review and consolidation of quality assessment. Pharmacoeconomics 2006;24:355-71.

25. Evers $\mathrm{S}$, Goossens $\mathrm{M}$, de Vet $\mathrm{H}$, et al. Criteria list for assessment of methodological quality of economic evaluations: consensus on health economic criteria. Int J Technol Assess Health Care 2005;21:240-5.

26. Jaime Caro J, Eddy DM, Kan H, et al. Questionnaire to assess relevance and credibility of modeling studies for informing health care decision making: an ISPOR-AMCP-NPC Good Practice Task Force report. Value Health 2014;17:174-82. 